\title{
Prevalencia del Síndrome de HELLP y sus resultados Perinatales en
} pacientes del Hospital Materno Infantil

C.E. ${ }^{1}$ Comité Editorial de la Revista de Postgrados de Medicina UNAH, Dirección de Docencia e Investigación-HE: Dr. Carlos Vargas Pineda, Dr. Rolando-Aguilera-L, Dra. Guadalupe-Romero-A, Julissa Yaneth Andrade Sanchez ${ }^{2}$, María Carolina Bustillo ${ }^{3}$

\section{Prevalence and perinatal results of HELLP syndrome in patients attended in the Delivery Room of Hospital Materno Infantil.}

Resumen

Objetivo:

Determinar la Prevalencia y los Resultados Perinatales del Síndrome de HELLP en las pacientes atendidas en la Sala de Labor y Parto del Hospital Materno Infantil

\section{Métodos:}

Se realizo un estudio descriptivo trasversal en la sala de labor y parto del Hospital Materno Infantil, que abarcó el periodo comprendido de julio 2008 a junio 2010. El universo de estudio estuvo constituido por 62 pacientes que cumplían con los criterios de inclusión, definidos por Sibai.

\section{Resultados:}

De los 62 casos encontrados con diagnostico de síndrome de HELLP, la edad media de prevalencia las pacientes fue entre 20-29 años (56.45\%), la mayoría el $59.7 \%$ se les diagnostico la patología estudiada en el puerperio inmediato dentro de los resultados perinatales el $12.91 \%$ al momento de interrumpir el embarazo eran obitos fetales, de los $87.09 \%$ recién nacidos vivo $16.7 \%$ tuvieron un puntaje de APGAR menor de 5 al primer minute de vida.

Conclusión: el síndrome de HELLP es una causa morbi-mortalidad materna y perinatal importante en nuestro país así como a nivel mundial

\section{Recomendación}

Tener una mejor clasificación del síndrome ya que hay un subregistro de casos, así como también tener una mejor comunicación con el servicio de medicina interna para el manejo en conjunto de las pacientes con esta patología. 


\section{Palabras clave: síndrome de HELLP, resultados perinatales, complicaciones. \\ Summary \\ Objetive}

Determine the prevalence and perinatal results of HELLP syndrome in patients attended in the Delivery Room of Hospital Materno Infantil.

\section{Methods}

It is a transversal descriptive study in the Delivery Room of Hospital Materno Infantil in the period from july 2008 to june 2010 . The universe were 62 patients that met inclusion criteria defined by Sibai.

\section{Results}

From the 62 casses with diagnosis of HELLP syndrome, the prevalence was in ages $20-29$ years (56.45\%), $59.7 \%$ the pathology was diagnosed in the puerperal period; on the perinatal outcomes, 12.91 were still births at the time of delivery, and of the $87.09 \%$ live newborns, $16.7 \%$ has and APGAR score less than five at the first minute.

CONCLUSION: HELLP syndrome is an important cause of maternal and perinatal morbidity and mortality.

\section{Recommendation}

Have a better classification of the Syndrome's pathology for there are nonregistered cases, as having a better communication with the service of Internal Medicine for the mutual management with the service of obstetrics and gynecology.

Key words

HELLP syndrome, perinatal outcomes, complications

\section{Introducción}

Los desordenes hipertensivos representan la complicación médica mas común en el embarazo afectando entre 6 y $8 \%$ de las gestaciones ${ }^{1}$. Dentro de las complicaciones de los desordenes hipertensivos, encontramos al síndrome de HELLP el cual fue descrito por primera vez en 1982 por Weinstein presentandose en el $0.17 \%$ al $0.85 \%$ de las gestaciones 2,3 .

Entre el $3.5 \%$ y el $10.5 \%$ de casos de muertes maternas a nivel mundial son a consecuencia del sindrome de HELLP, en Honduras 
para el 2008 la mortalidad maternal por transtornos hipertenivos fue de $21.89 \%^{4}$, también lleva al aumento de las tasas de morbimortalidad, tales como edema pulmonar (8\%), insuficiencia renal aguda(3\%), coagulación intravascular diseminada(15\%), desprendimiento placentario $\quad(9 \%), \quad$ ruptura hepática(1\%) , por lo que el reconocimiento temprano de esta afección permite adoptar conductas terapéuticas que lleven a un mejor pronóstico. $^{5}$

La tasa de mortalidad perinatal registradas oscilan entre $7.4 \%$ a $20.4 \%$ asociadas restricción del crecimiento fetal así como a la edad gestacional al momento del nacimiento por lo que los lleva a una alta tasa de complicaciones neonatales agudas entre ellas síndrome de dificultad respiratoria, displasia pulmonar, hemorragia intracerebral y enterocolitis necrotizante..$^{6,7,8}$

El diagnostico se establece de acuerdo a la hemólisis, la disfunción hepática y la trombocitopenia, criterios establecidos por Sibai y Martin $^{9,10}$.

El manejo del síndrome de HELLP es la evacuación inmediata del embarzo dándose solo un manejo conservador por 48-72 horas para cumplir inductores de madurez pulmonar en aquellos embarazos menores de 34 semanas de gestación, dentro de las terapias de manejo la Dexametasona $10 \mathrm{mg}$ de cada 12 horas sigue siendo la principal terapia de manejo ${ }^{11,12}$

En nuestro país existen pocos informes sobre el síndrome HELLP, lo que nos motivo a realizar la presente investigación con el proposito determinar la Prevalencia del síndrome de HELLP y sus resultados perinatales asi como determinar la prevalencia de complicaciones maternas y mortalidad en las pacientes embarazadas que son atendidas en a Sala de Labor y Parto del Hospital Materno Infantil durante.

\section{Materiales y Métodos}

Fue un estudio descriptivo trasversal. Las pacientes que se 
escogieron para este estudio se captaron atraves del sistema de estadística del Hospital Materno Infantil que se registraban con el diagnostico de síndrome de HELLP desde 1 de julio del 2008 al 30 de junio del 2010. El instrumento de recolección de datos fue por medio de una fuente secundaria ya que se realizó revisión de expedientes clinícos y se registrarón los datos en un instrumento de recopilación de información basado en apartados sociodemográficos generales (edad, ,procedencia), historia ginecoobstétrica, antecedentes personales patológicos, enfermedad actual, exámenes de laboratorio, embarazo actual, condición del recién nacido, tratamiento recibido, requerimiento de unidad de cuidados intensivos y disponibilidad de esta sala, así como casos de mortalidad materna.

El universo lo constituyeron el $100 \%$ que corresponde a 62 pacientes que cumplian con los criterios definidos por Sibai para síndrome de HELLP: Trombocitopenia definida como recuento plaquetario inferior a 100.000 por mm3.
Elevación de enzimas hepáticas dadas por AST mayor de $70 \mathrm{UI} / \mathrm{L}$.

Hemólisis definida por: Bilirrubina total mayor de $1.2 \mathrm{mg} / \mathrm{dl}$, Deshidrogenasa láctica mayor de $600 \mathrm{UI} / \mathrm{L} \mathrm{y} / \mathrm{o}$ frotis de sangre periférica compatible con hemólisis.

\section{Criterios de exclusión:}

pacientes que no cumplan con los criterios de síndrome de HELLP y que no se encontro el expediente en estadistica del sistema de registro del Hospital Escuela.

El análisis de la información se realizo a través del programa EPI INFO versión 3.4.3a Centers for disease control \& Prevention (C.D.C.) para el manejo de la base de datos y el procesamiento de la información, se sacarón, medidas de tendencia central, frecuencias y porcentajes.

Toda la información recavada proviene de datos obtenidos de los expedientes de cada paciente que fue diagnosticada con síndrome de HELLP, en el sistema de estadística del Hospital Materno Infantil.

\section{Resultados}


Durante el periodo de estudio entre 1 de Julio 2008 y 30 de Junio 2010, se encontraron registrados, 62 casos de síndrome de HELLP que cumplían con los criterios de inclusión.

Se observo que la edad media de las pacientes fue entre 20-29 años con 35 casos, con un porcentaje de $56.45 \%$, de los 30-39 años en numero de 18 con un porcentaje de $29.04 \%$, las de 40 años o más 4 casos con un porcentaje $6.45 \%$.

En el $59.7 \%$ se les diagnostico síndrome de HELLP en el puerperio inmediato (tabla 1).

Tabla 1. Momento de diagnostico del Síndrome de HELLP del 1 de Julio del 2008 al 30 de junio del 2010.

\begin{tabular}{|l|r|r|}
\hline $\begin{array}{l}\text { Momento del } \\
\text { diagnostico }\end{array}$ & Frecuencia & Porcentaje \\
\hline INGRESO & $\mathbf{2 5}$ & $\mathbf{4 0 . 3 \%}$ \\
\hline \begin{tabular}{l|r|r} 
PUERPERIO \\
INMEDIATO
\end{tabular} & $\mathbf{3 7}$ & $\mathbf{5 9 . 7 \%}$ \\
\hline Total & $\mathbf{6 2}$ & $\mathbf{1 0 0 . 0 \%}$ \\
\hline
\end{tabular}

Fuente: expedientes clínicos del sistema de registro del Hospital Materno Infantil

El grupo de pacientes multíparas fue más afectado en nuestra población estudiada 24 casos con un porcentaje de $38.7 \%$ y el $33.9 \%$ eran nuligestas.

Cabe mencionar que $38.71 \%$ de las pacientes fueron referidas de otras regiones hospitalaria principalmente de la región número 3 que corresponde al departamento de Comayagua en un $16.1 \%$.

Al estudiar los antecedentes personales, el $8.6 \%$ de las pacientes tenían antecedente de trastorno hipertensivo de base al momento del diagnostico de síndrome de HELLP, de estos $6.5 \%$ refirió que en sus embarazos anteriores había presentado preeclampsia.

Al momento del diagnostico del síndrome de HELLP el $58.06 \%$ de las pacientes tenían un embarazo entre 28-36 6/7 semanas de gestación y solo el $3.23 \%$ tenían entre 20-27 6/7 semanas de gestación.

$45.2 \%$ de las pacientes que presentarón complicaciones estan descritas en la ( tabla 2)

Tabla 2. Frecuencia de las complicaciones presentadas por las pacientes con Síndrome de HELLP 
atendidas en la Sala de Labor y Parto del Hospital Materno Infantil.

\begin{tabular}{|c|c|c|}
\hline Complicaciones & Frecuencia & Porcentaje \\
\hline Insuficiencia renal & 7 & $11.3 \%$ \\
\hline $\begin{array}{l}\text { Hematoma } \\
\text { hepático }\end{array}$ & 0 & $0 \%$ \\
\hline $\begin{array}{l}\text { Coagulación } \\
\text { intravascular } \\
\text { diseminada }\end{array}$ & 6 & $9.7 \%$ \\
\hline Neumonía & 6 & $9.7 \%$ \\
\hline $\begin{array}{l}\text { Desprendimiento } \\
\text { prematuro de } \\
\text { placenta }\end{array}$ & 7 & $11.3 \%$ \\
\hline Edema pulmonar & 2 & $3.2 \%$ \\
\hline Total & 28 & $45.16 \%$ \\
\hline
\end{tabular}

Fuente: expedientes clínicos del sistema de registro del Hospital Materno Infantil

De los resultados perinatales se encontró que de los 62 casos el12.91\% al momento de interrumpir el embarazo eran obitos fetales, de los $87.09 \%$ recién nacidos vivos $16.7 \%$ tuvieron un puntaje de APGAR menor de 5 al primer minuto de vida. en cuanto al peso del recién nacido $53.3 \%$ tenia un peso entre $1600-2500 \mathrm{~g}$ por lo que encontramos que la mayoria que fue ingresado a sala de neonatologia fue dado de alta $69.1 \%$ y 17 casos $30.9 \%$ fueron reportados como fallecidos

\section{Discusión}

La prevalencia del síndrome de HELLP en nuestro estudio fue de 62 casos en dos años de duración de la investigación.

El síndrome de HELLP es considerado uno de las principales complicaciones del embarazo que lleva a nivel mundial altas tasas de morbimortalidad materna y perinatal en nuestro estudio encontramos 6 casos de muerte materna que corresponde al $9.7 \%$ acorde con la literatura ${ }^{1,3}$

La edad de aparición según la literatura es de 20-29 años ${ }^{6}$ lo que coincide en nuestro estudio ya que $56.45 \%$ estaban en ese rango.

Asi también es mas frecuente en multiparas presentándose en este estudio $38.7 \%$ de los casos coincidente con otros estudios ${ }^{6}$

Suele ser diagnosticado antes del parto ${ }^{5}$ contrario a lo encontrado en este estudio que el $59.7 \%$ se diagnostico en el puerperio inmediato. 
Dentro de las complicaciones maternas al igual a la literatura la principal complicacion $^{7}$, igual a nuestro estudio es la insuficiencia renal y desprendimiento prematuro de placenta en un $11.3 \%$, coagulación intravascular diseminada y neumonia en 6 casos ( $9.7 \%)$ respectivamente.

Se registraron 6 casos(9.7) de muertes maternas directas por síndrome de HELLP.

Tambien cabe mencionar que de las pacientes ingresadas 9 requerian unidad de cuidados inmediatos y a ninguna de ellas se les traslado a dicha sala por la disponibilidad de cupo.

En cuanto a los resultados perinatales hubieron 8 casos de obitos fetales $12.91 \%$ y de los ingresados a sala de neonatologia fue dado de alta $69.1 \%$ y 17 casos $30.9 \%$ fueron reportados como fallecidos.

Como limitantes del estudio se tenemos que en primer lugar el servicio de estadistica del Hospital materno infantil tiene clasificado por el CIE-10 al síndrome de HELLP bajo el codigo de preeclampsia leve (014.0), así como también la poca colaboracion para poder acceder a los expedientes clínicos.

\section{Referencias}

1. Bujold, ms Emmanuel, Prevención de la Preeclampsia y Restricción del Crecimiento Intrauterino con aspirina empezar al comienzo del embarazo: un meta-análisis, Obstet Gynecol, 2010, vol. 116(402-403)

2. Navarro Vargas J. R., Bautista Charry A., Lozada Camacho J., Síndrome Hellp y Coagulopatía; Revista Venezolana de Anestesiología 2002; 7(2):pag.148.

3. Lopez Gomez, José Ramón, et al. Síndrome HELLP en la Maternidad del Hospital "Dr. Adolfo Prince Lara, 2001, vol.61, no.2, p.77-81.

4. Secretaria de salud, Defunciones en hospitales $y$ clinicas, departamento de estadística , cuadro n 210, 2008.

5. Sarabia Sánchez Eulalia, Gómez Díaz Julio, Morales García Víctor; Preclampsia Severa, Eclampsia, 
Síndrome de HELLP, Rev Fac Med UNAM 2005; 48(4): 145150.

6. Macchi Liliana Dora; Síndrome HELLP; Patología de Urgencia, Año 9, No. 2, Junio de 2001.

7. Romero Arauz, Juan Fernando; Lara González, Alma Lina; Ramos León Julio César; Izquierdo Puente Juan Carlos; Morbimortalidad materna en síndrome de HELLP; Ginecol. obstet. Méx; 69(5):189-193, mayo 2001.

8. Aguilar Nolvia; Síndrome HELLP estudio retrospectivo realizado en el hospital escuela, bloque materno infantil; Rev Med Post UNAH; 1998, volumen 3;pag 1-3.

9. Usandizaga R., Muñoz, E. Martín, M. de la Calle; El síndrome HELLP, Situación actual; Departamento de Obstetricia y Ginecología. Hospital Universitario La Paz. Madrid. Enero - Febrero 2002 Vol. XIV, № 1

10. Toirac Lamarque Alberto, Pascual López Viviana y Torres Gonzalez Yamilet, Síndrome de
Hellp, un síndrome o una entidad dentro de un síndrome, MEDISAN, 2002;6(2):68-79

11. Parra- Ramírez, Pablo y Beckles- Maxwell, Mario. Diagnóstico y Manejo Oportuno del Síndrome de HELLP. Acta méd. costarric, ene. 2005, vol.47, no.1, p.07-14. ISSN 0001-6002.

12.- Matchaba P, Moodley J. Corticoesteroides para el síndrome de HELLP en el embarazo (Cochrane Review). In: La Biblioteca Cochrane Plus, Issue 2, 2007. 